\title{
Active systemic lupus erythematosus is associated with a reduced cytokine production by $B$ cells in response to TLR9 stimulation
}

\author{
Julia Sieber ${ }^{1,2+}$, Capucine Daridon ${ }^{1,2+}$, Sarah J Fleischer ${ }^{1,2}$, Vanessa Fleischer ${ }^{1,2}$, Falk Hiepe ${ }^{1,2}$, Tobias Alexander ${ }^{1,2}$, \\ Guido Heine ${ }^{3}$, Gerd R Burmester', Simon Fillatreau ${ }^{2}$ and Thomas Dörner ${ }^{1,2^{*}}$
}

\begin{abstract}
Introduction: Systemic lupus erythematosus (SLE) is an autoimmune disease associated with a break in self-tolerance reflected by a production of antinuclear autoantibodies. Since autoantibody production can be activated via nucleic acid Toll-like receptor 9 (TLR9), the respective pathway has been implicated in the development of SLE and pathogenic $B$ cell responses. However, the response of B cells from SLE patients to TLR9 stimulation remains incompletely characterized.
\end{abstract}

Methods: In the current study, the response of B cells from SLE patients and healthy donors upon TLR9 stimulation was analyzed in terms of proliferation and cytokine production and correlated with the lupus disease activity and anti-dsDNA titers.

Results: B cells from SLE patients showed a reduced response to TLR9 agonist compared to B cells from healthy donors in terms of proliferation and activation. B cells from SLE patients with higher disease activity produced less interleukin (IL)-6, IL-10, vascular endothelial growth factor, and IL-1ra than B cells from healthy donors. Further analyses revealed an inverse correlation of cytokines produced by TLR9-stimulated B cells with lupus disease activity and anti-dsDNA titer, respectively.

Conclusion: The capacity of B cells from lupus patients to produce cytokines upon TLR9 engagement becomes less efficient with increasing disease activity, suggesting that they either enter an exhausted state or become tolerant to TLR stimulation for cytokine production when disease worsens.

\section{Introduction}

Systemic lupus erythematosus (SLE) is a severe systemic autoimmune disease with heterogeneous clinical manifestations [1]. A hallmark of SLE immunopathology is B-cell hyperactivity leading to increased numbers of circulating plasma cells [2] and a breakdown of self-tolerance toward DNA and nucleoproteins, which is reflected by elevated levels of antinuclear autoantibodies, such as antidouble-stranded (ds)DNA, anti-ribonucleoprotein and other autoantibodies [3]. In addition, SLE is associated

\footnotetext{
* Correspondence: thomas.doerner@charite.de

${ }^{\dagger}$ Equal contributors

'Department of Medicine/Rheumatology and Clinical Immunology, Charité University Medicine Berlin, CC12, Charitéplatz 01, 10098 Berlin, Germany ${ }^{2}$ German Rheumatism Research Center Berlin (DRFZ), a Leibniz Institute, Charitéplatz 01, 10098 Berlin, Germany

Full list of author information is available at the end of the article
}

with abnormal cytokine levels, including increased levels of type I interferon (IFN), IL-6, TNF- $\alpha$, and B-cell activating factor (BAFF), which are thought to have fundamental roles in the maintenance and progression of this inflammatory disease [4-12].

The role of B cells in immunity has been mainly related to the generation of antibodies and formation of immune complexes for a long period of time. However, $B$ cells can exert additional functions, such as antigen presentation, activation of $\mathrm{T}$ cells, formation of lymphoid organs and secretion of cytokines, but their contribution in human autoimmunity has not been comprehensively explored [13-16]. However, there is now clear evidence that cytokine-producing B cells can have important roles during autoimmune diseases, suggesting that the role of B cells in SLE pathogenesis might be extended beyond autoantibody production. 
It has been shown that cytokine production of B cells can be efficiently induced by toll-like receptor (TLR) signaling [17-19]. In this context, TLR9 is of great interest for SLE immunopathology because increased apoptosis and/or clearance deficiencies in SLE are considered to result in increased amounts of circulating plasma DNA, which may act as TLR agonists and subsequently provide B cell activation signals [20].

Earlier studies showed that SLE B cells responded in a similar way as healthy donors upon TLR9 stimulation. However, B cells from patients with severe SLE showed a reduced secretion of IL-6 and IL-10, and no upregulation of activation markers, such as CD86 after
TLR9 engagement compared to healthy donors [21,22]. To reconcile these findings, we undertook a more comprehensive study of cytokine production by B cells in SLE. The current study compared B cells from healthy donors and SLE patients for production of cytokines and growth factors, proliferation and expression of activation markers upon TLR9 stimulation taking the underlying lupus activity into consideration.

\section{Materials and methods}

\section{Patients and controls}

For the analysis of cytokine production by B cells, peripheral blood was collected from 18 SLE patients

Table 1 Demographic and clinical data, lupus activity (SLEDAI) and individual therapy of the patients at the time of analysis

\begin{tabular}{|c|c|c|c|c|c|c|c|}
\hline Patient ID & Sex & Age, y & B cells $/ \mu \mathrm{L}$ blood & anti-dsDNA IgG, U/mL & SLEDAI & Treatment & Prednisolone dose, $\mathrm{mg} / \mathrm{d}$ \\
\hline$\overline{\text { SLE1 }}$ & $F$ & 61 & 187 & 0 & 7 & Pred & 5.0 \\
\hline SLE2 & $\mathrm{F}$ & 31 & 320 & 0 & 5 & Pred, MTX & 5.0 \\
\hline SLE3 & $\mathrm{F}$ & 30 & 159 & 0 & 4 & Pred, MMF & 5.0 \\
\hline SLE4 & $\mathrm{F}$ & 25 & 132 & 45 & 8 & Pred, Aza, HCQ & 5.0 \\
\hline SLE5 & $\mathrm{F}$ & 36 & 216 & 38 & 8 & Pred, Aza & 7.5 \\
\hline SLE6 & $\mathrm{F}$ & 39 & 538 & 50 & 6 & Pred, MMF & 5.0 \\
\hline SLE7 & $\mathrm{F}$ & 22 & 164 & 0 & 4 & Pred & 5.0 \\
\hline SLE8 & $\mathrm{F}$ & 44 & 108 & 0 & 5 & $\mathrm{HCQ}$ & 0 \\
\hline SLE9 & $\mathrm{F}$ & 48 & 67 & 75 & 8 & Pred, MMF & 5.0 \\
\hline SLE10 & M & 20 & 89 & 50 & 10 & Pred, Aza & 5.0 \\
\hline SLE11 & $\mathrm{F}$ & 30 & 305 & 45 & 6 & Pred & 5.0 \\
\hline SLE12 & $\mathrm{F}$ & 33 & 468 & 0 & 5 & Pred & 8.0 \\
\hline SLE13 & $\mathrm{F}$ & 23 & 785 & 125 & 14 & Pred, HCQ & 100.0 \\
\hline SLE14 & $\mathrm{F}$ & 33 & 110 & 18 & 4 & Pred, HCQ & 5.0 \\
\hline SLE15 & $\mathrm{F}$ & 32 & 75 & 60 & 12 & Pred, MMF & 8.0 \\
\hline SLE16 & $\mathrm{F}$ & 37 & ND & 2,000 & 15 & Pred, HCQ & 25.0 \\
\hline SLE17 & $\mathrm{F}$ & 38 & 192 & 21 & 8 & Pred, HCQ, MMF & 7.5 \\
\hline SLE18 & $\mathrm{F}$ & 47 & 114 & 1,400 & 14 & Therapy naive & 0 \\
\hline SLE19 & $\mathrm{F}$ & 59 & 26 & 0 & 5 & Pred, Aza & 5.0 \\
\hline SLE20 & $\mathrm{F}$ & 30 & 32 & 1,000 & 5 & $\mathrm{HCQ}$ & 0 \\
\hline SLE21 & $\mathrm{F}$ & 51 & 81 & 200 & 6 & Pred, Aza & 7.5 \\
\hline SLE22 & $\mathrm{F}$ & 28 & 56 & 0 & 6 & Ciclosporine, Pred & 5.0 \\
\hline SLE23 & $\mathrm{F}$ & 33 & 98 & 0 & 6 & Pred, Aza & 5.0 \\
\hline SLE24 & $\mathrm{F}$ & 32 & 155 & 17.5 & 18 & Cylophosphamide, Pred & 7.5 \\
\hline SLE25 & $\mathrm{F}$ & 29 & 19 & positive & 7 & Ciclosporine, Pred & - \\
\hline SLE26 & M & 21 & 79 & positive & 6 & Pred, MMF & - \\
\hline SLE27 & $\mathrm{F}$ & 37 & 118 & positive & 5 & Pred, Aza & - \\
\hline SLE28 & $\mathrm{F}$ & 74 & 20 & negative & 5 & Antimalarials & - \\
\hline SLE29 & $\mathrm{F}$ & 35 & 75 & positive & 6 & Pred, Antimalarials & - \\
\hline SLE30 & $\mathrm{F}$ & 33 & 35 & positive & 7 & Pred, MMF & - \\
\hline
\end{tabular}

Patient SLE18 was newly diagnosed and donated blood before immunosuppressive treatment was started. SLEDAl, systemic lupus erythematosus disease activity index; Pred, prednisolone; Aza, azathioprine; MTX, methotrexate; HCQ, hydroxychloroquine; MMF, mycophenolate mofetil; ND, not detected. 


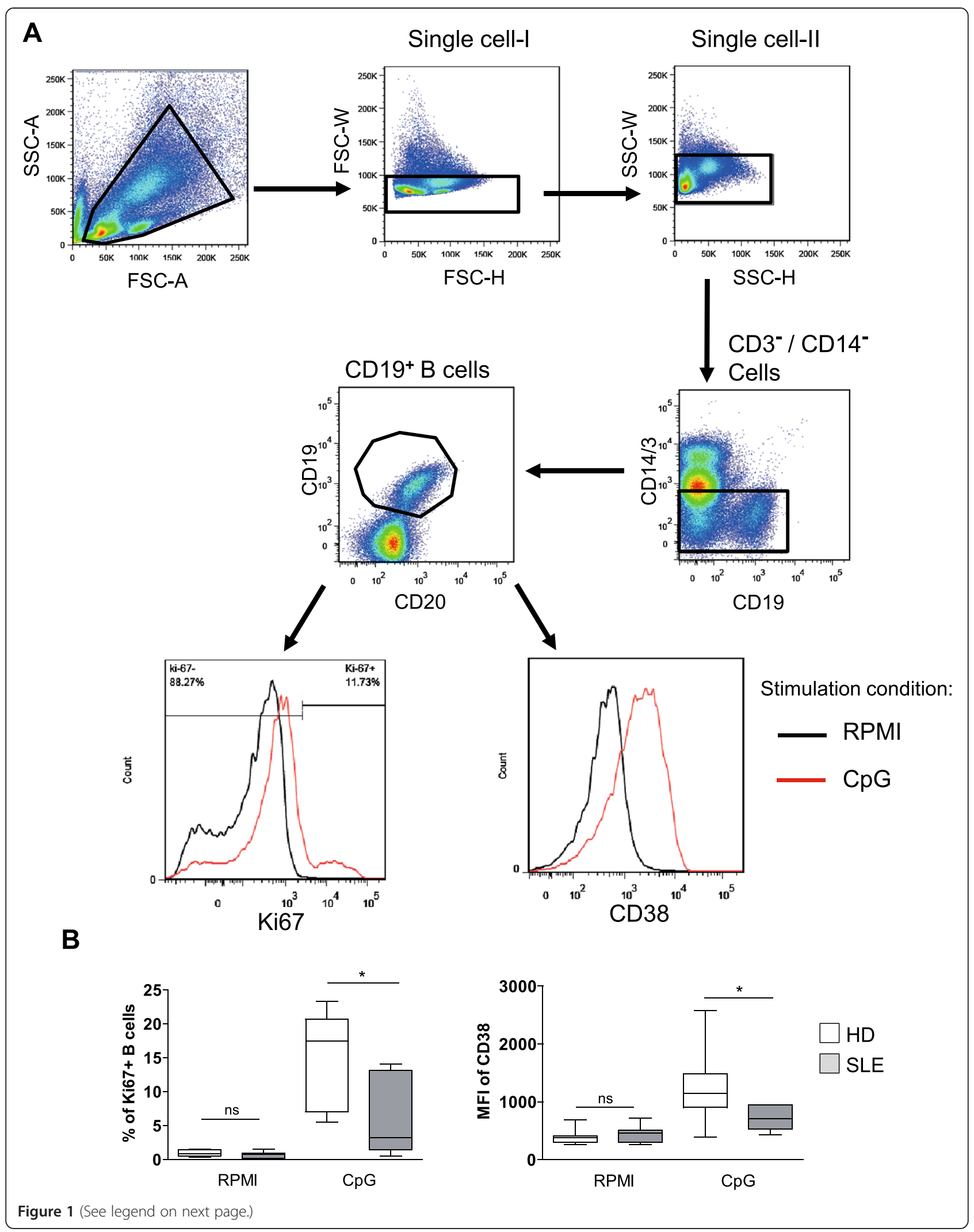


(17 females/1 male) with a mean age of $34.9 \pm 10.4$ years and 13 healthy donors (12 females/1 male) with a mean age of $36.7 \pm 14.9$ years. For the analysis of activation and IL-10 expression in B cells using flow cytometry (FC), peripheral blood was collected from 6 female SLE patients with a mean age of $38.8 \pm 12.9$ years and 10 healthy donors ( 8 female/2 male) with a mean age of $32.9 \pm 11.1$ years. For the analysis of TLR9 expression, peripheral blood was collected from patients with SLE (12 female/ 1 male, $38.4 \pm 18.4$ ) and 5 female healthy donors $(29.4 \pm 5.0)$.

The study was approved by the local ethics committee of the Charité Universitätsmedizin Berlin and written consent was obtained from all donors. The consents are on file held by the principal investigator and available for review by the editor-in-chief upon request. All patients met the revised American College of Rheumatology classification criteria for SLE [23]. The disease activity was assessed using the SLE disease activity index (SLEDAI) modified according to the SELENA-trial [24]. Details of the clinical characteristics and treatment regimens of the analyzed SLE patients are provided in Table 1.

\section{Isolation of B cells}

Peripheral blood mononuclear cells (PBMCs) were isolated with density gradient centrifugation using lymphocyte separation medium (PAA Laboratories, Pasching, Austria) as previously described [25]. Subsequently, B cells were negatively purified by magnetic activated cell sorting $\left(\mathrm{MACS}^{\circ}\right)$ using the B-cell Isolation Kit II (Miltenyi Biotec, Bergisch Gladbach, Germany) according to the manufacturer's instructions and B cell purity was checked by flow cytometry. The contamination with $\mathrm{CD}^{+}, \mathrm{CD}^{+} 4^{+}$ and dead cells was below $5 \%$ in all samples.

\section{Lymphocyte staining for flow cytometry}

Purified B cells were stained at $4^{\circ} \mathrm{C}$ for 15 minutes with antibodies against CD14-PB (M5E2), CD3-PB (UCHT1), CD27-Cy5 (2E4), CD19-PE-Cy7 (SJ25C1), CD20-PerCPCy5 (L27), and IgD-FITC (IA62) to control the purity used for the subsequent analyses.

Before and after stimulation, PBMCs were stained first with antibodies against CD14- Pacific blue (PB) (M5E2), CD3-PB (UCHT1), CD27-fluorescein isothiocyanate (FITC) (L128), CD38-PercP-Cy5.5 (HIT2) and CD20-Pacific orange (PO) (H147) for 10 minutes on ice. After washing,
PBMCs were incubated with $400 \mu \mathrm{l}$ of $1 \times$ FACS permeabilizing solution 2 (Becton Dickinson (BD) Franklin Lakes, NJ, USA) for 10 minutes at room temperature (RT). After permeabilization and washing, PBMCs were stained with anti-Ki67-PE-Cy7 (B56) and anti-IL-10-APC (JES39D7) and anti-TLR9-PE (eB72-1665) antibodies for $10 \mathrm{mi}-$ nutes at RT. All antibodies were purchased from BD; beside Cy5-conjugated anti-CD27 antibody (2E4) (kind gift from Andreas Thiel, Berlin Center for Regenerative Therapy, Charité Berlin) and anti-IL-10-APC antibodies purchased from Miltenyi Biotec. Stained cells were analyzed by FC using the FACSCanto ${ }^{\mathrm{m}}$ II flow-cytometer (BD). FC data were analyzed using FlowJo (Tree Star, Inc., Ashland, OR, USA).

\section{In vitro stimulation}

B cells were stimulated in vitro with CpG 2006 oligonucleotide (CpG) (TIB MolBiol Synthese Labor GmbH, Berlin, Germany). The cells were resuspended in RPMI 1640 Glutamax supplemented with 10\% FCS (Lonza, Köln, Germany), 5\% penicillin/streptomycin, and $0.05 \mathrm{mM}$ 2-mercaptoethanol (Gibco $^{\circ}$ Life Technologies $\mathrm{GmbH}$, Darmstadt, Germany). B cells, $10^{5}$, were seeded and stimulated with $2.5 \mu \mathrm{g} / \mathrm{mL}$ CpG for $48 \mathrm{~h}$ at $37^{\circ} \mathrm{C}$ and $5 \% \mathrm{CO}_{2}$. After 2 days of culture, the supernatants were harvested and frozen at $-70^{\circ} \mathrm{C}$ prior to analysis.

To analyze the IL-10 production by $\mathrm{B}$ cells, PBMCs $\left(10^{6} /\right.$ well $)$ were cultured with CpG 2006 in vitro as described $[26,27]$. Intracellular staining of IL-10 and Ki67 was performed on PBMCs after 2 days of culture. PBMCs were re-stimulated for $4 \mathrm{~h}$ with $10 \mathrm{ng} / \mathrm{mL}$ PMA and $1 \mu \mathrm{M}$ ionomycin including $2 \mu \mathrm{g} / \mathrm{mL}$ brefeldin A for the last $2 \mathrm{~h}$ (all from Sigma Munich, Germany) prior to intracellular staining. Unstimulated cells served as controls.

\section{Cytokine assay}

Cryopreserved supernatants were assessed for determination of cytokine concentration using Bio-Plex technology (Bio-Rad Laboratories, Inc., CA, USA) according to the manufacturer's instructions. The cytokines analyzed were IL-1 $\beta$, IL-1ra, IL-2, IL-4, IL-5, IL-6, IL-7, IL-8/ CXCL8 (chemokine (C-X-C motif) ligand 8), IL-9, IL-10, IL-12p70, IL-13, IL-15, IL-17A, eotaxin-1/CCL11 (chemokine (C-C motif) ligand 11), basic fibroblast growth factors (FGF), granulocyte colony-stimulating factor (GCSF), granulocyte macrophage colony-stimulating factor (GM-CSF), IFN- $\alpha 2$, IFN- $\gamma$, IP-10 (IFN $\gamma$-induced protein 10)/CXCL10, monocyte chemotactic protein-1 (MCP-1)/ 
CCL2, macrophage inflammatory protein-1 $\alpha$ (MIP-1 $\alpha) /$ CCL3, MIP-1 $\beta / C C L 4$, platelet-derived growth factor-BB (PDGF-BB), regulated on activation, normal T-cell expressed and secreted (RANTES)/CCL5, vascular endothelial growth factor (VEGF) and TNF- $\alpha$. The assay sensitivity depends on the particular cytokines analyzed from $0.3 \mathrm{pg} / \mathrm{mL}$ for IL-10 to $6.4 \mathrm{pg} / \mathrm{mL}$ for IFN- $\gamma$. Although all 28 cytokines and growth factors were detectable in the supernatant of the B-cell culture; 12 of them (IL-1 3 , IL-2, IL-4, IL-5, IL-7, IL-12p70, IL-13, IL-15, GM-

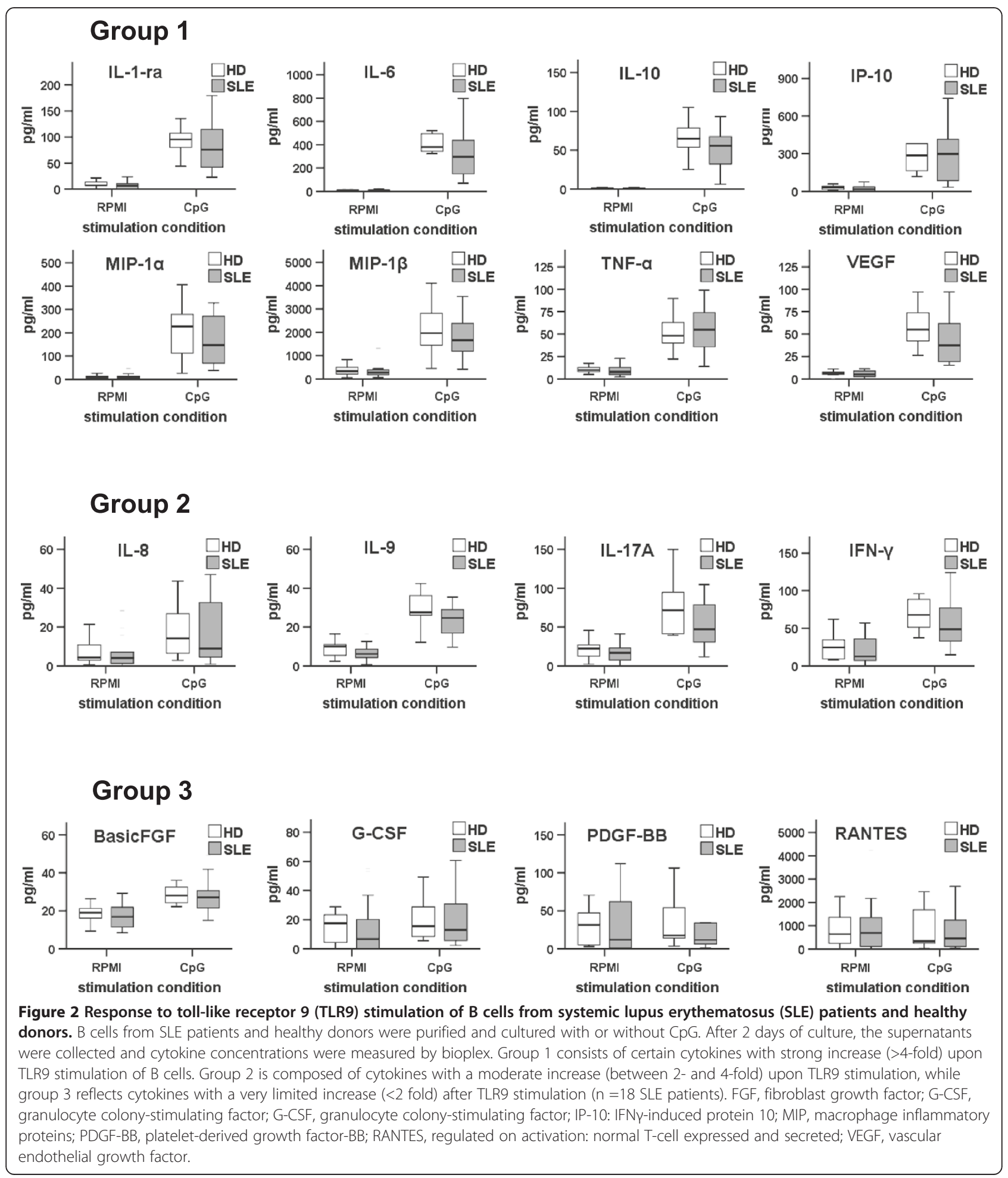


A

Patient No. Concentration of cytokines in the supernatant after TLR9 stimulation SLEDAI IL-1raIL-6 IL-8 IL-9 IL-10 IL-17A IFN- $y$ IP-10 MIP-1 $\alpha$ MIP-1 $\beta$ TNF- $\alpha$ VEGF

SLE7

SLE14

SLE2

SLE8

SLE12

SLE6

SLE11

SLE1

SLE4

SLE5

SLE9

SLE17

SLE10

SLE15

SLE13

SLE18

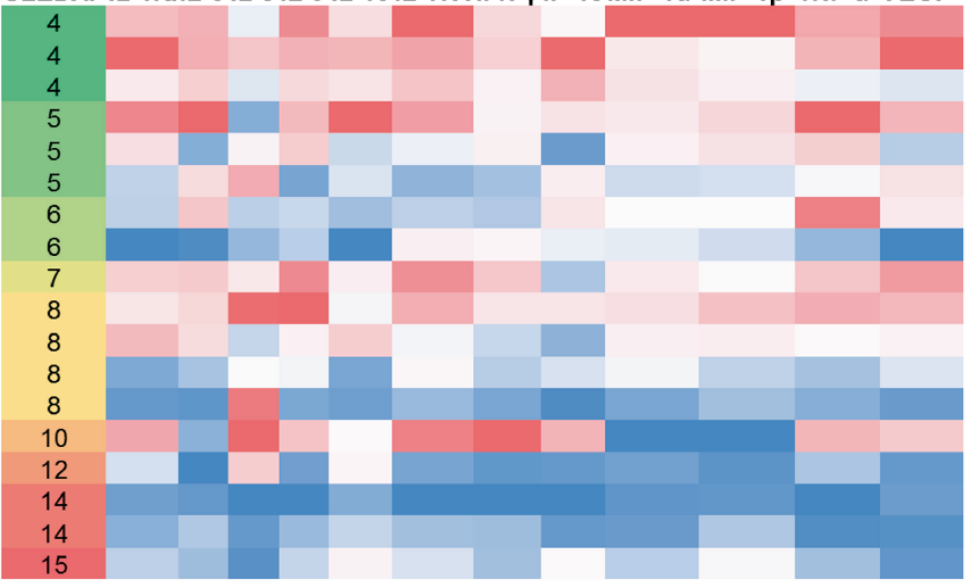

SLE16
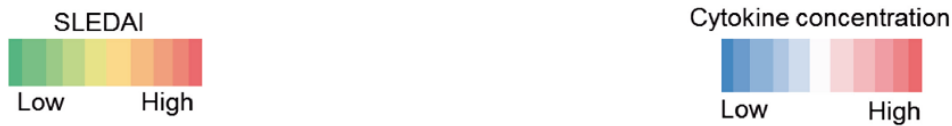

B

Inverse correlation with the lupus disease activity (SLEDAI)
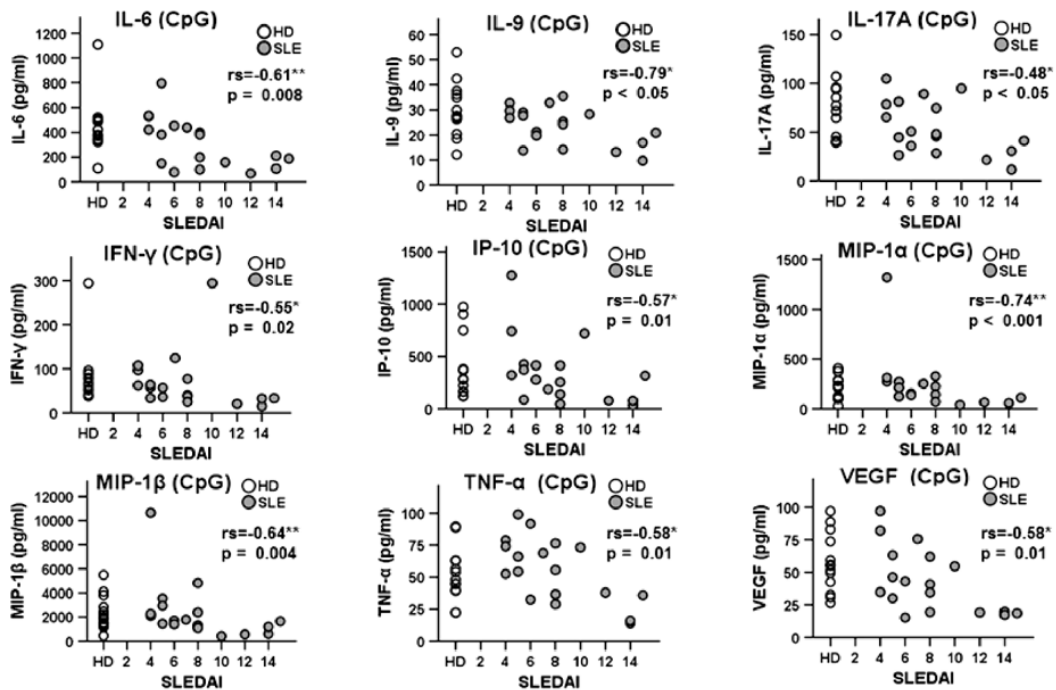

No correlation with the lupus disease activity (SLEDAI)
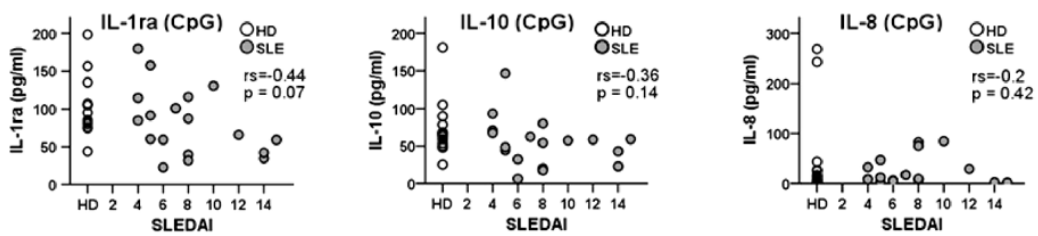

Figure 3 (See legend on next page.) 
(See figure on previous page.)

Figure 3 Hyporesponsiveness to toll-like receptor 9 (TLR9) stimulation of B cells from systemic lupus erythematosus (SLE) patients in relation to their disease activity. (A) Heat map of cytokines from group 1 and 2 secreted by B cells upon TLR9 stimulation (dark blue for the lowest, to red for the highest concentration of cytokine in the supernatant). SLE patients were ordered according to their disease activity from low systemic lupus erythematosus disease activity index (SLEDAI) in green, to high SLEDAI in red. (B) Direct correlation between individual cytokines from group 1 and 2 and lupus activity (SLEDAI score). Significant inverse correlation was found between the SLEDAI score and individual cytokines produced by B cells upon TLR9 stimulation (IL-6, IL-9, IL-17A, IFN- $\gamma$, IP-10, MIP-1a, MIP-1 $\beta$, and TNF-a). Healthy donors (HD) are plotted as reference. (Spearman $r$ correlation test; ${ }^{*} P<0.05$ and ${ }^{* *} P<0.01$ ). IP-10: IFNY-induced protein 10; MIP, macrophage inflammatory proteins; VEGF, vascular endothelial growth factor.

CSF, IFN- $\alpha 2$, eotaxin-1, and MCP-1) were produced at low levels with a mean concentration below $20 \mathrm{pg} / \mathrm{mL}$ after TLR9 stimulation (Additional file 1) and were therefore not considered for further analysis.

\section{Statistical analysis}

The statistical analysis was performed with SPSS (version 20, IBM, NY, Chicago, IL, USA). To compare data from healthy donors and SLE patients, the nonparametric MannWhitney $U$-test was used. The Wilcoxon test was used to compare results after TLR9 stimulation with unstimulated controls for the cytokine production. Multiple comparisons were performed using one way analysis of variance (ANOVA) with Dunnett's post hoc test. To correlate cytokine levels with SLEDAI scores or with dsDNA titers, Spearman correlation analysis was performed. P-values $<0.05$ were considered statistically significant. The statistical tests used are indicated in each figure legend.

\section{Results}

In the current study, a comprehensive analysis was performed to assess the capacity of B cells from SLE patients to respond to TLR9 stimulation in terms of proliferation, activation, and cytokine production in relation with clinical lupus activity using SLEDAI.

\section{B cells from SLE patients have a reduced proliferation and activation upon TLR9 stimulation}

We first evaluated the response of B cells to TLR9 stimulation in terms of proliferation and activation. The frequency of proliferating cells ( $\%$ of $\mathrm{Ki}^{+} 7^{+} \mathrm{B}$ cells), and the upregulation of the activation marker CD38 after 2-day culture with CpG were analyzed by FC (Figure 1A). CpG induced B cell proliferation independently of B cell receptor (BCR) engagement; however, SLE patients had a lower frequency of proliferating B cells upon TLR9 stimulation in comparison with healthy donors $(P<0.05)$ (Figure $1 \mathrm{~B}$, left graph). Moreover, activation of B cells was evaluated by upregulation of CD38 expression (mean fluorescence intensity, MFI) upon TLR9 stimulation. B cells from healthy donors significantly upregulated CD38, resulting in a 3-fold increase after TLR9 stimulation, while the response of $B$ cells from SLE patients was significantly lower (Figure 1B, right graph, $P<0.05$ ).

\section{B cells from SLE patients secrete fewer cytokines in relation to the disease activity}

Subsequently, the influence of TLR9 stimulation on cytokine production was evaluated by analyzing the concentrations of 28 cytokines and growth factors in the supernatants of B cell cultures from healthy donors and SLE patients using BioPlex technology. The cytokines were grouped according to the level of their induction upon TLR9 engagement compared to unstimulated B cells. The first group included cytokines showing more than a 4-fold increase after TLR9 stimulation and comprised IL-1ra, TNF- $\alpha$, IL-6, and IL-10, the chemokines IP-10, MIP-1 $\alpha,-1 \beta$, and the growth factor VEGF. The second group was defined by a moderate (2- to 4-fold) increase after TLR9 stimulation, and comprised IL-8, IL-9, IL-17A and IFN- $\gamma$. A third group of growth factors and chemokines, defined by a very small increase or no increase after TLR9 stimulation (maximum 2-fold increase) comprised basic FGF, G-CSF, PDGF-BB, and RANTES (Figure 2). Overall, the profiles of cytokine secretion observed in the supernatants of cultured B cells from SLE patients and healthy donors shared very large similarities. We found that none of the cytokines from groups 1 and 2 were secreted at a higher level by B cells from SLE patients, but rather at lower levels in comparison to B cells from healthy donors. Since earlier reports found that B cells from active SLE patients were less responsive to TLR9 stimulation in terms of IL-6 and IL-10 production [21], we analyzed the relation between the cytokines from groups 1 and 2 and the disease activity (SLEDAI) by using a heat map (Figure 3A). When the patients were ordered according to their SLEDAI, it became apparent that B cells from patients with SLEDAI of $4(n=3)$ produced larger amounts of cytokines than those from patients presenting with a SLEDAI higher than $14(n=3)$. The remaining patients with a SLEDAI between 4 and 14 displayed an intermediate but clearly ranked profile (Figure 3A). In greater detail, correlation analyses of individual cytokines revealed significant inverse correlations between the SLEDAI and inducible 
amounts of IL-6, IL-9, IL-17A, IFN- $\gamma$, IP-10, MIP-1 $\alpha$, MIP-1 $\beta$, TNF- $\alpha$, and VEGF of TLR9-activated SLE B cells (Figure $3 \mathrm{~B}$ ). In contrast, there was no correlation between the spontaneous (unstimulated) production of these cytokines and the SLEDAI, highlighting the specificity of this association with TLR9 signaling.

A

Concentration of cytokines in the supernatant after TLR9 stimulation, CpG
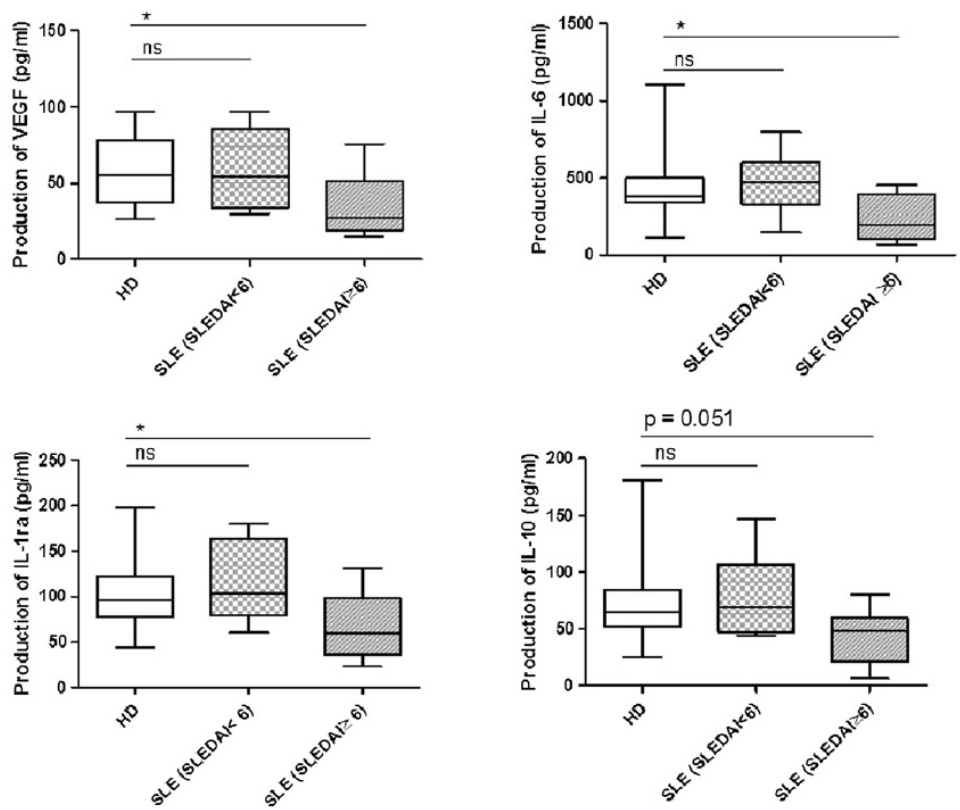

B

No stimulation, RPMI
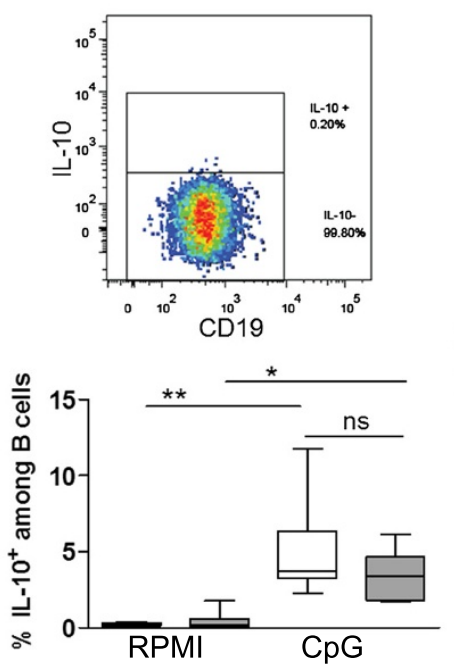

TLR9 stimulation, $\mathrm{CpG}$

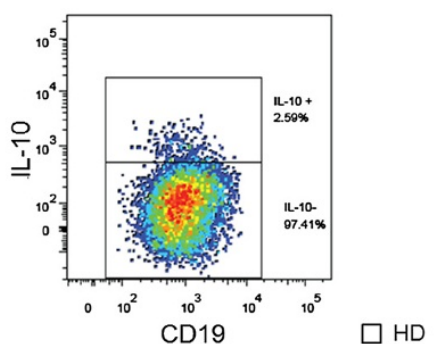

$\square$ SLE

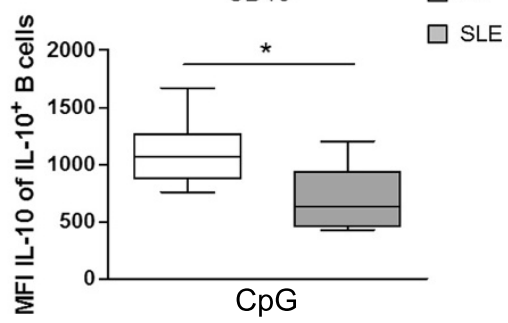

Figure 4 Reduced IL-6, vascular endothelial growth factor (VEGF), and IL-1ra production by B cells upon toll-like receptor 9 (TLR9) stimulation in active systemic lupus erythematosus (SLE) patients compared to healthy donors (HD). (A) To compare the production of cytokines (group 1 and 2) upon TLR9 stimulation by healthy donors with SLE patients, the SLE cohort was divided in two groups with low disease activity (SLEDAI $<6, n=6$ ) and high disease activity (SLEDAI $\geq 6, n=12$ ). IL-6, VEGF, and IL-1 ra produced by B cells upon TLR9 stimulation were significantly reduced in SLE with high SLEDAI compared to healthy donors (one way analysis of variance with Dunnett's post hoc test, *P <0.05). (B) Representative flow cytometry analysis plots showing IL-10-producing B cells after 2 days of peripheral blood mononuclear cells (PBMC) culture without (left) or with (right) CpG stimulation. Combined data from 6 SLE patients and 10 healthy controls for the frequency of IL-10-producing B cells (left graph) and the overall production of IL-10 by B cells (right graph, MFI = mean fluorescence intensity, reflecting amount per cell) (Mann-Whitney $U$-test and Wilcoxon test; ns: not significant, ${ }^{*} P<0.05$, ${ }^{* *} P<0.01$ ). 
This global analysis showed that TLR9-stimulated B cells from patients with active SLE produced fewer cytokines than those from patients with less active disease. In order to further compare the data to healthy donors, we divided the patients into two groups with high $(\geq 6)$ and low SLEDAI $(<6)$ [28] (Figure 4A). Notably, the production of IL-6, IL-1ra, and VEGF by B cells was significantly reduced in patients with active SLE with a SLEDAI $\geq 6$ compared to healthy donors. The secretion of IL-10 was also reduced by trend, although not statistically significant $(P=0.051)$. FC analysis of IL-10-producing B cells showed a significantly increased frequency of IL- $10^{+} \mathrm{B}$ cells after TLR9 stimulation (Figure 4B). However, there was no difference between B cells from SLE patients and healthy donors. While the generation of IL-10-producing B cells was not reduced in culture of B cells from SLE patients, there was a clear reduction of the amount of IL-10 induced in individual IL- $10^{+} \mathrm{B}$ cells compared to healthy donors as shown by the MFI of intracellular IL-10 (Figure 4B).

\section{Inverse correlation between cytokine production and} anti-dsDNA autoantibodies

A key serologic parameter reflecting the breakdown of tolerance and related with lupus disease activity are antidsDNA autoantibodies that have been linked to TLR9 stimulation [29]. We found a significant $(P<0.05)$ inverse correlation between the serum anti-dsDNA titers and amounts of IL-1ra, IL-6, IL-9, IL-17A, IFN- $\gamma$, MIP-1 $\alpha,-1 \beta$, TNF- $\alpha$, and VEGF produced by TLR9-activated B cells from SLE patients (Figure 5). Thus, the higher antidsDNA antibody titer in serum, the lower was the level

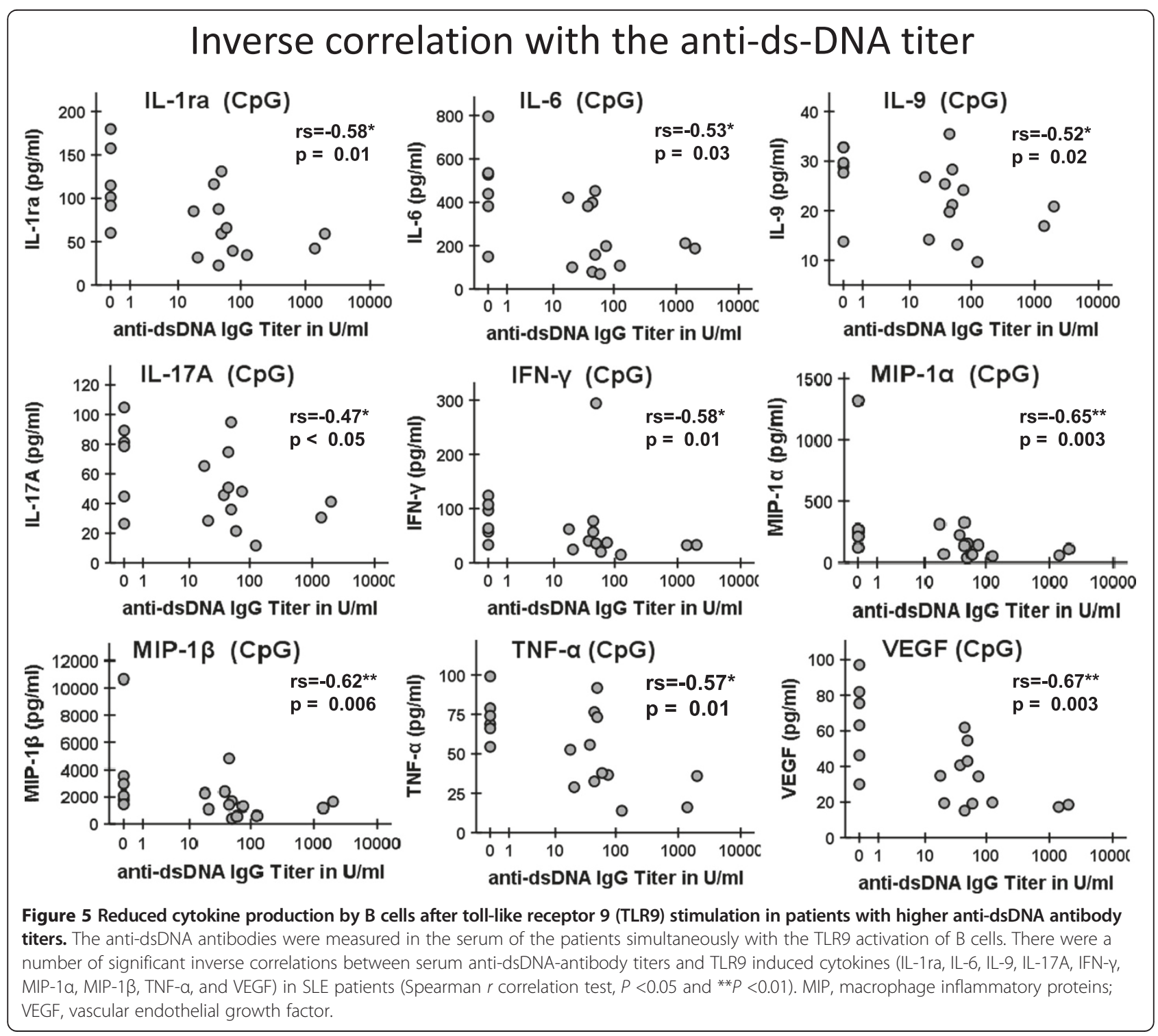


of these cytokines produced in vitro by $\mathrm{CpG}$ activated B cells.

\section{TLR9 is downregulated in patients with active SLE}

In an attempt to explain the reduced response of B cells from SLE patients to TLR9 agonist compared to controls, we analyzed whether TLR9 was differentially expressed by these cells. FC analysis of TLR9 expression in B cells from healthy donors and SLE patients showed that the MFI of TLR9 was similar in B cells from healthy donors compared to those from SLE patients $(242.8 \pm 41.9$ and $218.5 \pm 53.2)$ (Figure 6). However, a significant reduction of TLR9 expression was found for B cells from SLE patients with a SLEDAI $\geq 6(191.8 \pm 19.5)$ (Figure 6). This result is in line with our observations on cytokine production, suggesting that the observed reduction in $\mathrm{CpG}$ responsiveness for $\mathrm{B}$ cells from patients with high disease activity could be related to a down-modulation of TLR9 expression.

\section{Discussion}

Our results confirm that B cells from SLE patients globally show the same pattern of cytokine expression compared to B cells from healthy donors upon TLR9 stimulation [30]. However, our study also provides evidence that B cells from patients with severe SLE are hyporesponsive to TLR9 stimulation in terms of activation, proliferation, and cytokine production compared with B cells from healthy donors. Moreover, the reduction of cytokine production upon TLR9 stimulation was correlated with lupus activity and
anti-ds-DNA antibody titers. Patients with high SLEDAI score (SLEDAI 26 ) showed a lower secretion of IL-6, IL-1ra, IL-10, and VEGF upon TLR9 stimulation in comparison to healthy donors as well as a lower expression of TLR9. Of note, a previous report documented that SLE B cells expressed significantly more TLR9 than B cells from healthy donors [31], especially in patients with increased anti-dsDNA antibody titers and high disease activity $[32,33]$. Although the reason for the discrepancy between our results and this study remains unexplained, it seems coherent that B cells from patients with active SLE have both reduced TLR9 expression and a lower response to TLR9 agonist than B cells from healthy donors. Remarkably, we did not identify any cytokine that was induced to a larger extent in B cells from SLE patients compared to healthy donors.

In this study the mechanism responsible for lower expression of TLR9 by B cells from patients with active SLE remains to be delineated. A possibility is that $B$ cells from patients with severe SLE are hyporesponsive to TLR9 stimulation because of an overstimulation in vivo by circulating DNA in the serum of the patients [21]. This might indicate an exhausted or post-activation state as already described for T cells from SLE patients [1], or a state of tolerance to TLR-stimulation as described for myeloid cells [34]. Another reason might be that TLR9 signaling limits the life span of anti-DNA B cells, leading to an elimination of the B cells expressing high amounts of TLR9, as shown in an SLE mouse model [35]. In any case, such a

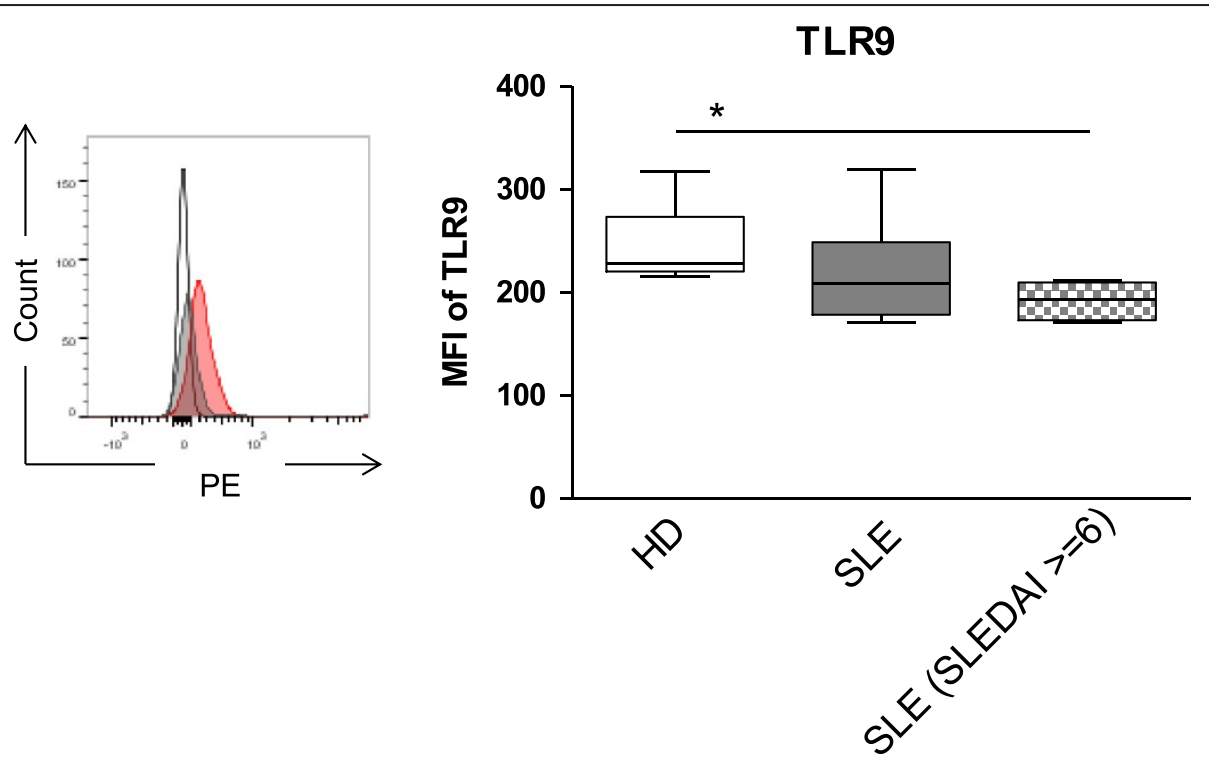

Figure 6 Reduced expression of toll-like receptor 9 (TLR9) by B cells from active systemic lupus erythematosus (SLE) patients.

Representative flow cytometry histogram showing TLR9 staining for B cells (left). Combined data for TLR9 detection of B cells obtained from 6 SLE patients and 4 healthy donors (HD), respectively, did not show a significant difference in TLR9 detection, but B cells from patients with more active SLE (SLE disease activity index (SLEDAI) $\geq 6$ ) were found to express reduced TLR9 (Mann-Whitney U-test; ns: not significant; $\left.{ }^{*} P<0.05\right)($ right panel). Hhistogram: red line TLR9, gray line isotype- phycoerythrin (PE) and black line control antibody; MFI: mean fluorescence intensity of TLR9. 
loss of TLR9 responsiveness might represent an attempt of the immune system to reduce the availability of this potentially deleterious pathway as the disease worsens. Alternatively, intrinsic TLR9 signaling in B cells might be beneficial in SLE so that the observed impairment could play a role in exacerbation of the disease. It is currently difficult to evaluate whether intrinsic TLR9 signaling in B cells is beneficial or deleterious during chronic SLE. TLR9stimulated B cells secreted inflammatory cytokines, such as IL-6, TNF- $\alpha$, MIP- $1 \alpha,-1 \beta$, and IP-10, but also antiinflammatory cytokines such as IL-1ra [36], and IL-10 $[13,37]$. We found reduced IL-10 production by B cells upon TLR9 stimulation for active SLE patients, consistent with previous reports [21]. IL-1ra and VEGF have also been described to have immunosuppressive effects, and were also produced in lower amounts by B cells from active patients compared to healthy donors. Indeed, IL-1ra is a receptor antagonist that inhibits the IL-1 pathway and provides an important anti-inflammatory mechanism [38]. VEGF has also been described as an immunosuppressive cytokine that inhibits the functional maturation of dendritic cells and T-cell development [39,40]. The link between SLE pathogenesis and the reduced expression of IL-1ra and VEGF by B cells remains to be further analyzed.

The capacity of B cells to produce cytokines can be influenced by a number of variables in addition to the disease activity, including immune therapies. In this regard, the current data do not indicate that certain therapies (Table 1) may have major effects on cytokine-production by B cells upon stimulation in vitro. In particular there was no significant difference in the amount of cytokines produced by $B$ cells from patients treated with hydroxychloroquine $(n=6)$ or not $(n=12)$ (data not shown), although this drug is considered to inhibit TLR9-signaling [41]. We also correlated the dosage of prednisolone $(\mathrm{mg} /$ day) taken by the patient at the moment of the study and the cytokine level produced by B cells upon TLR9 stimulation (data not shown). Only the concentration of IL-9, IL-17A, IFN- $\gamma$ and RANTES showed a significant inverse correlation with the dosage of prednisolone used by the patients. Nevertheless, only two patients received more than $10 \mathrm{mg}$ prednisolone/ day in this study. In addition, one newly diagnosed and untreated patient (SLE18) with a SLEDAI of 14 showed a very low cytokine production, in a range comparable to the other patients with active SLE who were under immunosuppressive treatment. Thus, the described low cytokine production upon TLR9 stimulation by B cells of active patients seems related to SLE disease activity per se rather than to immunosuppressive interventions.

\section{Conclusion}

The cytokine production by B cells from patients with severe SLE upon TLR9-engagement ex vivo is substantially lower than in healthy donors. The current data are consistent with an exhaustion of B cells, or an induction of TLR-tolerance post-activation (by diminished TLR9 expression) depending on lupus disease activity. Understanding the molecular mechanism of reduced cytokine production by B cells upon TLR9 engagement in SLE might provide new insights into the pathogenesis of SLE.

\section{Additional file}

\begin{abstract}
Additional file 1: Figure S1. Cytokines lacking substantial production upon toll-like receptor 9 (TLR9) stimulation by B cells from healthy donors (white) and patients suffering from systemic lupus erythematosus (SLE) (gray). B cells from SLE-patients and healthy donors were purified and cultured with or without $\mathrm{CpG}$, the cytokine concentrations were measured in supernatants collected after 2 days of culture by bioplex. The cytokines shown were detectable at very low levels after B-cell stimulation by TLR ligation using $\mathrm{CpG}(<20 \mathrm{pg} / \mathrm{mL}$ of supernatant).
\end{abstract}

\section{Abbreviations}

Aza: azathioprine; BAFF: B-cell activating factor; Basic FGF: fibroblast growth factors; BCR: B cell receptor; BD: Becton Dickinson; CCL: chemokine (C-C motif) ligand; CXCL: chemokine (C-X-C motif) ligand; FC: flow cytometry; FITC: fluorescein isothiocyanate; G-CSF: granulocyte colony-stimulating factor; GM-CSF: granulocyte macrophage colony-stimulating factor; HCQ: hydroxychloroquine; IFNm: interferon; IL: interleukin; IP-10: IFNY-induced protein 10; MCP-1: monocyte chemotactic protein-1; MIP: macrophage inflammatory proteins; MMF: mycophenolate mofetil; MTX: methotrexate; PB: Pacific blue; PBMCs: peripheral blood mononuclear cells; PDGF-BB: plateletderived growth factor-BB; PE-Cy: phycoerythrin-cyanin; PerCP: peridininchlorophyll protein; PO: Pacific orange; Pred: prednisolone; RANTES: regulated on activation, normal T-cell expressed and secreted; SLE: systemic lupus erythematosus; SLEDAl: systemic lupus erythematosus disease activity index; TLR9: toll-like receptor 9; TNF: tumor necrosis factor; VEGF: vascular endothelial growth factor.

\section{Competing interests}

The authors declare that they have no competing interest.

\section{Authors' contributions}

TD and CD designed the study. JS, VF, SJF and CD carried out experimental work in different areas. SJF, VF, TA, GRB, GH, SF and FH discussed the data at several stages and worked on the manuscript. CD, JS, SJF, SF and TD analyzed data. All authors helped to draft the manuscript, read and approved the final manuscript.

\section{Acknowledgements}

The authors would like to thank Karin Reiter for technical support and all the patients and healthy donors who contributed to the study. Written informed consent was obtained from patients and controls based on the approval of the local ethics committee, including for publication of data. The consent form is held by the principal investigator and is available for review by the editor-in-chief upon request. The study has been supported by grants of the Deutsche Forschungsgemeinschaft (DFG) with grants of the SFB650 and SFB633, SPP Immunobone (Do491/8-2) and individual projects Do491/7-2,3.

\section{Author details}

'Department of Medicine/Rheumatology and Clinical Immunology, Charité University Medicine Berlin, CC12, Charitéplatz 01, 10098 Berlin, Germany. ${ }^{2}$ German Rheumatism Research Center Berlin (DRFZ), a Leibniz Institute, Charitéplatz 01, 10098 Berlin, Germany. 'Department of Dermatology, Venerology and Allergology, Allergy-Center-Charité, Charité University Medicine Berlin, Luisenstraße 2, 10117 Berlin, Germany.

Received: 9 May 2014 Accepted: 22 October 2014

Published online: 11 November 2014 
References

1. Tsokos GC: Systemic lupus erythematosus. N Engl J Med 2011, 365:2110-2121.

2. Jacobi AM, Odendahl M, Reiter K, Bruns A, Burmester GR, Radbruch A, Valet G, Lipsky PE, Dorner T: Correlation between circulating CD27high plasma cells and disease activity in patients with systemic lupus erythematosus. Arthritis Rheum 2003, 48:1332-1342.

3. Wahren-Herlenius M, Dorner T: Immunopathogenic mechanisms of systemic autoimmune disease. Lancet 2013, 382:819-831.

4. Grondal G, Gunnarsson I, Ronnelid J, Rogberg S, Klareskog L, Lundberg I: Cytokine production, serum levels and disease activity in systemic lupus erythematosus. Clin Exp Rheumatol 2000, 18:565-570.

5. Aringer M, Stummvoll GH, Steiner G, Koller M, Steiner CW, Hofler E, Hiesberger H, Smolen JS, Graninger WB: Serum interleukin-15 is elevated in systemic lupus erythematosus. Rheumatology 2001, 40:876-881.

6. Aringer M, Feierl E, Steiner G, Stummvoll GH, Hofler E, Steiner CW, Radda I, Smole JS, Graninger WB: Increased bioactive TNF in human systemic lupus erythematosus: associations with cell death. Lupus 2002, 11:102-108.

7. Blomberg S, Eloranta ML, Magnusson M, Alm GV, Ronnblom L: Expression of the markers BDCA-2 and BDCA-4 and production of interferon-alpha by plasmacytoid dendritic cells in systemic lupus erythematosus. Arthritis Rheum 2003, 48:2524-2532

8. Pers JO, Daridon C, Devauchelle V, Jousse S, Saraux A, Jamin C, Youinou P. BAFF overexpression is associated with autoantibody production in autoimmune diseases. Ann NY Acad Sci 2005, 1050:34-39.

9. Petri M, Stohl W, Chatham W, McCune WJ, Chevrier M, Ryel J, Recta V, Zhong J, Freimuth W: Association of plasma B lymphocyte stimulator levels and disease activity in systemic lupus erythematosus. Arthritis Rheum 2008, 58:2453-2459.

10. Ronnblom L, Pascual V: The innate immune system in SLE: type I interferons and dendritic cells. Lupus 2008, 17:394-399.

11. Obermoser $G$, Pascual V: The interferon-alpha signature of systemic lupus erythematosus. Lupus 2010, 19:1012-1019.

12. Munroe ME, Vista ES, Guthridge JM, Thompson LF, Merrill JT, James JA: Pro-inflammatory adaptive cytokines and shed tumor necrosis factor receptors are elevated preceding systemic lupus erythematosus disease flare. Arthritis Rheumatol 2014, 66:1888-1899.

13. Fillatreau S, Sweenie CH, McGeachy MJ, Gray D, Anderton SM: B cells regulate autoimmunity by provision of IL-10. Nat Immunol 2002, 3:944-950.

14. Barr TA, Shen P, Brown S, Lampropoulou V, Roch T, Lawrie S, Fan B, O'Connor RA, Anderton SM, Bar-Or A, Fillatreau S, Gray D: B cell depletion therapy ameliorates autoimmune disease through ablation of IL-6-producing $B$ cells. J Exp Med 2012, 209:1001-1010.

15. Lund FE: Cytokine-producing B lymphocytes-key regulators of immunity. Curr Opin Immunol 2008, 20:332-338.

16. Anolik JH: B cell biology: implications for treatment of systemic lupus erythematosus. Lupus 2013, 22:342-349.

17. Agrawal S, Gupta S: TLR1/2, TLR7, and TLR9 signals directly activate human peripheral blood naive and memory $B$ cell subsets to produce cytokines, chemokines, and hematopoietic growth factors. J Clin Immunol 2011, 31:89-98.

18. Klinman DM, Yi AK, Beaucage SL, Conover J, Krieg AM: CpG motifs present in bacteria DNA rapidly induce lymphocytes to secrete interleukin 6 , interleukin 12, and interferon gamma. Proc Natl Acad Sci USA 1996 93:2879-2883.

19. Vollmer J, Jurk M, Samulowitz U, Lipford G, Forsbach A, Wullner M, Tluk S, Hartmann H, Kritzler A, Muller C, Schetter C, Krieg AM: CpG oligodeoxynucleotides stimulate IFN-gamma-inducible protein-10 production in human B cells. J Endotoxin Res 2004, 10:431-438.

20. Celhar T, Magalhaes R, Fairhurst AM: TLR7 and TLR9 in SLE: when sensing self goes wrong. Immunol Res 2012, 53:58-77.

21. Zorro S, Arias M, Riano F, Paris $S$, Ramirez LA, Uribe $O$, Garcia LF, Vasquez G Response to ODN-CpG by B Cells from patients with systemic lupus erythematosus correlates with disease activity. Lupus 2009, 18:718-726.

22. Zeuner RA, Klinman DM, Illei G, Yarboro C, Ishii KJ, Gursel M, Verthelyi D: Response of peripheral blood mononuclear cells from lupus patients to stimulation by CpG oligodeoxynucleotides. Rheumatology 2003, 42:563-569.

23. Hochberg MC: Updating the American College of Rheumatology revised criteria for the classification of systemic lupus erythematosus. Arthritis Rheum 1997, 40:1725.
24. Petri M, Kim MY, Kalunian KC, Grossman J, Hahn BH, Sammaritano LR, Lockshin M, Merrill JT, Belmont HM, Askanase AD, McCune WJ, Hearth-Holmes M, Dooley MA, Von Feldt J, Friedman A, Tan M, Davis J, Cronin M, Diamond B, Mackay M, Sigler L, Fillius M, Rupel A, Licciardi F, Buyon JP, OC-SELENA Trial: Combined oral contraceptives in women with systemic lupus erythematosus. N Engl J Med 2005, 353:2550-2558.

25. Mei HE, Yoshida T, Sime W, Hiepe F, Thiele K, Manz RA, Radbruch A, Dorner T: Blood-borne human plasma cells in steady state are derived from mucosal immune responses. Blood 2009, 113:2461-2469.

26. Heine $G$, Niesner U, Chang HD, Steinmeyer A, Zugel U, Zuberbier T, Radbruch A, Worm M: 1,25-dihydroxyvitamin D(3) promotes IL-10 production in human B cells. Eur J Immunol 2008, 38:2210-2218.

27. Heine G, Drozdenko G, Grun JR, Chang HD, Radbruch A, Worm M: Autocrine IL-10 promotes human B-cell differentiation into IgM- or IgG-secreting plasmablasts. Eur J Immunol 2014, 44:1615-1621.

28. Wang G, Pierangeli SS, Papalardo E, Ansari GA, Khan MF: Markers of oxidative and nitrosative stress in systemic lupus erythematosus: correlation with disease activity. Arthritis Rheum 2010, 62:2064-2072.

29. Chen $M$, Zhang W, Xu W, Zhang F, Xiong S: Blockade of TLR9 signaling in $B$ cells impaired anti-dsDNA antibody production in mice induced by activated syngenic lymphocyte-derived DNA immunization. Mol Immunol 2011, 48:1532-1539.

30. Wong CK, Wong PT, Tam LS, Li EK, Chen DP, Lam CW: Activation profile of Toll-like receptors of peripheral blood lymphocytes in patients with systemic lupus erythematosus. Clin Exp Immunol 2010, 159:11-22.

31. Papadimitraki ED, Choulaki C, Koutala E, Bertsias G, Tsatsanis C, Gergianaki I, Raptopoulou A, Kritikos HD, Mamalaki C, Sidiropoulos P, Boumpas DT: Expansion of toll-like receptor 9-expressing B cells in active systemic lupus erythematosus: implications for the induction and maintenance of the autoimmune process. Arthritis Rheum 2006, 54:3601-3611.

32. Mu R, Sun XY, Lim LT, Xu CH, Dai CX, Su Y, Jia RL, Li ZG: Toll-like receptor 9 is correlated to disease activity in Chinese systemic lupus erythematosus population. Chin Med J (Engl) 2012, 125:2873-2877.

33. Chauhan SK, Singh W, Rai R, Rai M, Rai G: Distinct autoantibody profiles in systemic lupus erythematosus patients are selectively associated with TLR7 and TLR9 upregulation. J Clin Immunol 2013, 33:954-964.

34. Liew FY, Xu D, Brint EK, O'Neill LA: Negative regulation of toll-like receptor-mediated immune responses. Nat Rev Immunol 2005, 5:446-458,

35. Nickerson KM, Christensen SR, Cullen JL, Meng W, Luning Prak ET, Shlomchik MJ: TLR9 promotes tolerance by restricting survival of anergic anti-DNA B cells, yet is also required for their activation. J Immunol 2013, 190:1447-1456.

36. Arend WP: Interleukin 1 receptor antagonist. A new member of the interleukin 1 family. J Clin Invest 1991, 88:1445-1451.

37. Mauri C, Gray D, Mushtaq N, Londei M: Prevention of arthritis by interleukin 10-producing B cells. J Exp Med 2003, 197:489-501.

38. Arend WP: The balance between IL-1 and IL-1Ra in disease. Cytokine Growth Factor Rev 2002, 13:323-340.

39. Ohm JE, Gabrilovich DI, Sempowski GD, Kisseleva E, Parman KS, Nadaf S, Carbone DP: VEGF inhibits T-cell development and may contribute to tumor-induced immune suppression. Blood 2003, 101:4878-4886.

40. Angeli V, Ginhoux F, Llodra J, Quemeneur L, Frenette PS, Skobe M, Jessberger R, Merad M, Randolph GJ: B cell-driven lymphangiogenesis in inflamed lymph nodes enhances dendritic cell mobilization. Immunity 2006, 24:203-215.

41. Kuznik A, Bencina M, Svajger U, Jeras M, Rozman B, Jerala R: Mechanism of endosomal TLR inhibition by antimalarial drugs and imidazoquinolines. J Immunol 2011, 186:4794-4804.

doi:10.1186/s13075-014-0477-

Cite this article as: Sieber et al: Active systemic lupus erythematosus is associated with a reduced cytokine production by $B$ cells in response to TLR9 stimulation. Arthritis Research \& Therapy 2014 16:477. 